\title{
Measurements of flows over isolated valleys
}

\author{
Brian Garvey \\ Department of Geography, University of Sheffield, Sheffield, \\ SO10 2TN, UK \\ and School of Engineering Sciences, University of Southampton, Southampton, \\ SO17 1BJ, UK \\ Ian P Castro \\ School of Engineering Sciences, University of Southampton, Southampton, \\ SO17 1BJ, UK \\ Giles Wiggs \\ Department of Geography, University of Sheffield, Sheffield, SO10 2TN, UK \\ Joanna Bullard \\ Department of Geography, Loughborough University, Loughborough, \\ LE11 3TU, UK
}

November 2004

Abstract. Wind tunnel measurements of the flow over an isolated valley both normal and at an angle $\left(45^{\circ}\right)$ to a simulated neutrally stable atmospheric boundary layer are presented. Attention is concentrated on the nature of the near-surface flow upwind and downwind of the valley and the character of the flow within the valley itself. The work formed part of a wider study which included detailed field measurements around an African desert valley and some limited comparisons with that work are included. A scale of about 1:1000 was used for the laboratory work, in which an appropriate combination of hot wire and particle image velocimetry was employed. For a valley normal to the upwind flow, it is shown that the upstream influence of the valley extends to a distance of at least one half of the axial valley width upstream of the leading edge, whereas differences in mean flow and turbulence could be identified well beyond two valley widths from the downwind edge. Nonnormal wind angles lead to significant along-valley flows within the valley and, even at two valley heights above the valley ridge level, there remains a significant spanwise flow component. Downwind turbulence levels are somewhat lower in this case, but are still considerably higher than in the undisturbed boundary layer. At both flow angles, there are significant recirculation regions within the valleys, starting from mean separation just beyond the leading edge, but the strong spanwise flow in the $45^{\circ}$ case reduces the axial extent of the separated zone. The flow is shown to be in some ways analogous to flow over an isolated hill. Our results usefully enhance the field data and could be used to improve modelling of saltation processes in the field.

Keywords: Isolated valleys, field measurements, wind tunnel. $\mathrm{AT}_{\mathrm{EX}}$

(C) 2004 Kluwer Academic Publishers. Printed in the Netherlands. 


\section{List of Symbols}

$\begin{array}{ll}h & \text { Height of the Irwin spires } \\ H & \text { Valley height, mm } \\ L & \text { Valley width (i.e. in axial direction, see figure 1), } \\ & \text { measured between the leading and trailing edges at } \\ & \text { ridge height } \\ & \text { Valley width, measured as above but for approach } \\ & \text { flow at } 45^{\circ}\left(L^{\prime}=\sqrt{2} L\right) \\ L^{\prime} & \text { Surface shear velocity } \\ u_{*} & \text { The three normal turbulent stresses and the domi- } \\ u^{2}, \overline{v^{2}}, \overline{w^{2}},-\overline{u w} & \text { nant shear stress } \\ U_{r} & \text { Measured free-stream velocity, m/s } \\ U, V, W & \text { Mean velocities in the } x, y, z \text { directions } \\ x, y, z & \text { Cartesian coordinates, measuring axial, spanwise and } \\ & \text { vertical distance from the leading edge of the valley } \\ z^{\prime} & \text { Vertical distance above the (local) surface of the } \\ & \text { valley } \\ z_{o} & \text { Surface roughness length } \\ \delta & \text { Boundary layer height, measured to the } 98 \% \text { velocity } \\ \text { point } & \text { von Karman's log-law constant } \\ \kappa & \text { rms values of fluctuating velocities in the } x \text { and } z \\ \sigma_{u}, \sigma_{w} & \text { directions, respectively }\end{array}$

\section{Introduction}

\subsection{BACKGRound AND Motivation}

There is a substantial literature on the wind flow over isolated hills and many general features of such flow (e.g. speed-up at the crest) are well known. Isolated valleys in otherwise homogeneously flat surroundings are perhaps less common than isolated hills but they occur frequently in desert situations and there is clear evidence that their influence on the surrounding terrain - e.g. on the morphology of sand dunes - can be significant (see Weber \& Kauffman, 1998, for example). In contrast to the situation for hills, little is known about the effects of the valley on mean wind and turbulence structure both upwind and downwind, as well as within the valley itself, and there has never been an adequate field study to identify the effects of isolated valleys in flat, 
plateau terrain on boundary layer winds. Sierputowski et al (1995) and Snyder et al (1991) have reported practically the only relevant wind tunnel studies. The former is complicated by the fact that the valley was formed between two hills, so the flow field within the valley was undoubtedly affected by that over the upstream hill. It is in that sense typical of a number of studies of flow over sequences of hills (which could equally be viewed as sequences of valleys). Some of these have been undertaken specifically to assess the adequacy of theoretical and/or numerical studies of such 'topographically periodic' flows (as in Hunt et al, 1988), but they are of little help in identifying how an isolated valley affects the flow upstream and downstream.

There have been studies in other contexts of flow over incisions in an otherwise flat surface. Keough \& Addison (1994), for example, made observations in a water flume of the flow over a backward facing step with a downwind forward facing step - a 'cavity'. They found that the behaviour within the cavity was strongly dependent on its aspect ratio (axial length to depth ratio), with a recirculation zone confined to the first part of the cavity if this aspect ratio exceeded about 10. They applied their results to floodplain dynamics and pollution dispersal in rivers. Likewise, in engineering contexts there has been considerable work on flow in cavities; Shankar \& Deshpande (2000) provide a fairly recent discussion. In none of these cases, however, has the emphasis been on how the cavity affects the upstream and downstream flow - in particular, on how surface stresses and other near-wall features of the boundary layer are affected by the presence of the cavity. There have also been numerous studies of density stratified flow over valleys (e.g. Kimura \& Manins, 1988, and others reviewed in Baines, 1995). Again, the emphasis has been on flow within the valley, rather than outside it. And, finally, recent work in the urban meteorology field has included studies of street canyon flows, which might be thought of as cavity flows (e.g. Louka et al, 2000). Britter \& Hanna (2003) have provided a review but here, too, the situations are usually much more complex, geometrically, being characterised by consecutive rows of buildings and streets.

In the work to be reported in this paper, our major aim is to explore the details of the near-surface flow not just within an isolated valley, but to significant distances upwind and downwind. The work formed part of a larger programme, which included field studies in central Namibia, whose general aim was to explore the effect of an isolated valley on the geomorphology of the surrounding plane. Changing patterns of sand dunes have been associated with river valley systems (e.g. Lancaster, 1988, Bullard \& Nash, 1998), but no detailed studies of dune/valley interactions have previously been made, although it was clear from the 
earlier work that changes to the wind flow upstream and downstream of a valley, because of its presence, could clearly lead to changes in dune morphology, as noted above. These changes most commonly occur, not unnaturally, in relatively strong, neutrally stable wind conditions and it is that case that we consider here. The emphasis is on the characteristics of the flow itself. Implications for sand dune development and behaviour will be more fully explored elsewhere (initial work is reported in Garvey et al, 2004).

As implied above, the experiments of Snyder et al (1991), initially reported by Khurshudyan et al (1990) and with data subsequently archived in smoothed form by Busuoli et al (1993), is really the only previous relevant and well-controlled wind tunnel study. It was quite extensive and provides cleaner idealisations than the Sierputowski et al (1995) experiment since the valleys were genuinely sunk beneath the surface level of the upstream (and downstream) boundary layer. However, the emphasis in that work was on the effects of the valley on dispersion from elevated sources so there was relatively limited analysis of the flow and turbulence characteristics, particularly either upstream or downstream of the valley. It also differs from the present work in that the cross-sectional valley shapes were quite smooth. This meant that for the steepest valley, steady separation took place downwind of the leading edge. In the present work, we concentrate on a case in which there is a more abrupt change in surface slope at the leading edge, common in desert situations, so that separation occurs there. Furthermore, the valley shape was purposely chosen as an idealisation of the field case and our studies include a case when the valley axis is not normal to the approach flow. Nonetheless, it is appropriate here to summarise the findings of Snyder et al (1991) as far as the flows themselves are concerned, and this is done in the following section. Subsequently, the present field situation is outlined and then, in Section 3 , we provide details of the model valley, the wind tunnel set up and the techniques for the present experiments, along with the characteristics of the simulated atmospheric boundary layer. Section 4 presents the major results and includes comparisons with the Snyder et al (1991) data where appropriate. Final discussion and conclusions are given in Section 5 .

\subsection{The Snyder et al (1991) Study}

Snyder et al (1991) used three smoothly-shaped valleys of fixed depth $H$ - some $12 \%$ of the upstream boundary layer height, $\delta$, or about $730 z_{o}$, where $z_{o}$ is the roughness length of the undisturbed, upstream boundary layer. Valley widths in the axial (wind) direction were 6, 10 
and $16 \mathrm{H}$ and the respective maximum slopes were $26^{\circ}, 16^{\circ}$ and $10^{\circ}$. The results showed that only within the steepest valley was there a mean separated flow; this extended from a little way beyond the leading edge to some $4 \mathrm{H}$ downstream - i.e. beyond the valley centreline. Not surprisingly, turbulence stress levels were high within the separated shear layer forming the upper boundary of this region and remained high for some distance downwind of the valley's trailing edge. At six valley heights downstream of the trailing edge (i.e. one valley width downstream), for example, there was still a clear signature of the reattached mixing layer, with a peak turbulence shear stress some $30 \%$ higher than its value in the equilibrium upstream boundary layer. Even twice as far downstream the stresses remained measurably higher than those in the reference flow. The increases in turbulence stresses were naturally significantly smaller for the other two valleys, in which mean separation zones did not exist. Nonetheless, even for the widest valley, the shear stress at the valley centreline had a peak some $60 \%$ higher than the maximum value far upstream (but note that this is about five times lower than the peak for the steepest valley). Even for the steepest valley the effects of the valley on the downstream flow are significantly smaller than in the present case, as discussed later.

Upstream of the steepest valley, there were significant decreases in turbulence shear stress in the near surface region so that, at the leading edge for example, its value was only a little more than one half of its value far upstream. The behaviour in the near surface region was, however, strongly dependent on the shape of the valley. The widest valley led to an increase in near surface shear stress, of almost exactly the same magnitude as the decrease in the case of the narrowest valley. Only minor changes from the upstream flow were observed for the medium width valley. These various detailed results were neither presented nor discussed in the original paper and for the purposes of the present paper have been extracted from the smoothed data files subsequently prepared by Busuoli et al (1993). Comparisons with the present results made later in this paper are almost entirely on the basis of the data contained graphically in this latter report and available quantitatively on the internet.

\subsection{THE FIELD EXPERIMENT}

The valley shape used for the present wind tunnel work was an idealisation of a desert valley in central Namibia. This was 20-22 m deep and was virtually straight over at least $300 \mathrm{~m}$. Within this section the valley's width $(L)$ varied between 150 and $175 \mathrm{~m}$ at the edges and it had steep side slopes of $20-25^{\circ}$. The field experiments are fully 
described elsewhere (Garvey, 2003, Wiggs et al, 2002, Garvey et al, 2004). Trials were performed for situations which included cases when the upstream wind was either roughly normal to the valley axis or around $45^{\circ}$ to it. All the field data were collected during daylight hours, so in some cases the flows were, not surprisingly, convectively unstable. However, the intention was specifically to study relatively strong wind conditions (since these are the most important for aeolian transport). In all experiments the upwind temperature profile was monitored, allowing Richardson numbers to be estimated, and the comparisons made here with wind tunnel data are for cases in which the boundary layer was closely neutral. The best estimate for the roughness length for the upstream surface layer in such cases was $6.5 \mathrm{~mm}$ - deduced by using sonic anemometry measurements of turbulence shear stress, $-\overline{u w}$, to provide a (surrogate for) the friction velocity $u_{*}$ and ensuring the loglaw fit to the mean velocity data had the appropriate slope $\left(u_{*} / \kappa\right)$. The ratios of valley height to roughness height and boundary layer height were thus, respectively, $H / z_{o}=3200$ and $H / \delta=0.026$, assuming a neutral boundary layer height of about $800 \mathrm{~m}$, estimated from the usual relation, $\delta=u_{*} / 6 f$, where $f$ is the Coriolis parameter, $2 \Omega \sin (\phi)$ (with $\Omega$ and $\phi$ being the earth's rotation rate and the latitude, respectively). The valley aspect ratio, defined as the ratio of its width (normal to its axis) and its depth, was around $L / H=8$.

\section{Experimental Details}

\subsection{TEChNiques}

All the experiments were undertaken in the $2.3 \times 1.7 \mathrm{~m}$ closed return wind tunnel in the School of Engineering Sciences at the University of Southampton. This has a working section some $4.4 \mathrm{~m}$ in length and a free stream turbulence intensity below $0.4 \%$. The model valley (see fig.1) was positioned in an appropriate slot cut in one of the ground sections, at a location where its leading edge was some $3.4 \mathrm{~m}$ downstream of the working section entrance. For the experiments using a valley whose axis was at $45^{\circ}$ to the upstream flow, a second model was constructed, with exactly the same cross-stream dimensions (normal to its axis) as in fig.1, but inserted into another appropriately cut ground section, again with the leading edge (at the spanwise centre-line) positioned at the same downstream location as for the $0^{\circ}$ case. In both cases the entire valley surfaces were covered in the same coarse sandpaper used for the surrounding terrain (see Section 3.2). It should be noted that the spanwise aspect ratio of the valleys, defined as the ratio of the 
valley's spanwise length to its height, was about 70 (and about 100 in the $45^{\circ}$ case). The spanwise ends of the valleys were blocked off when they reached the working section's corner fillets. Possible consequencies of the three-dimensional flows near the ends are not considered too significant, since even the spanwise aspect ratio of the separated region (for the normal valley case) was around 12.5.

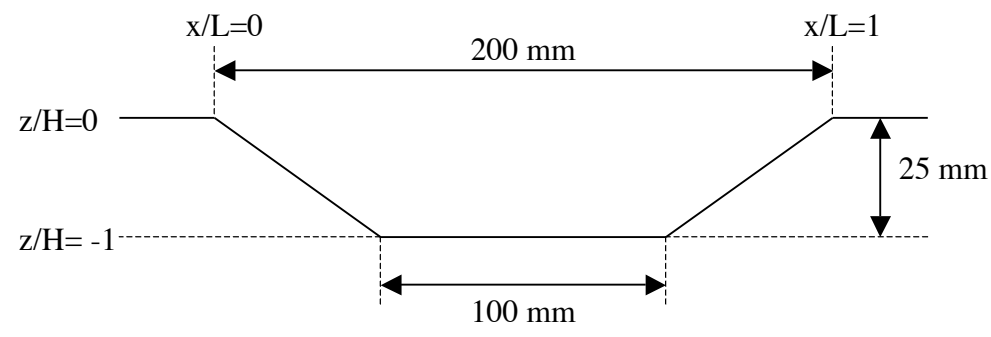

Figure 1. Idealised model valley for the wind tunnel experiment.

Measurements were made using an appropriate combination of standard crossed hot wire anemometry (HWA) and particle image velocimetry (PIV) techniques. For the former, the two gold-plated tungsten wires of 2.5 or $5 \mu \mathrm{m}$ diameter and an active length of about $1 \mathrm{~mm}$ and at nominally $\pm 45^{\circ}$ to the free stream direction were driven by bridges (from the University of Newcastle, N.S.W., Australia) using an overheat ratio of 1.5. Bridge outputs were amplified, filtered to prevent aliasing and digitally sampled at typically $2 \mathrm{kHz}$ via a 16 -bit $\mathrm{A} / \mathrm{D}$ convertor (ADC 488/8SA). All sampling and data collection for both calibration and subsequent measurement was controlled using a virtual instrument package written in LabVIEW (from National Instruments) and running on an Apple Macintosh computer. A pitot-static tube was mounted in the free stream and used to monitor tunnel speed, nominally $30 \mathrm{~m} \mathrm{~s}^{-1}$ for all data presented herein, and as a reference for wire calibration. Yaw calibration was done using the effective cosine method. In all profiles obtained, the initial free-stream measurement point was repeated at the end of the profile and if drift in mean velocity exceeded about $1 \%$ the profile was repeated after re-calibration of the wires.

For measurements within the valley, where turbulence intensities precluded effective use of HWA, a (Dantec UK) PIV system, with FlowManager 3.61 for image cross-correlation and deduction of resulting velocity vectors, was used to obtain 500 images at a sampling rate of 2 Hz. An 80C60 HiSense CCD camera with a 60 or $105 \mathrm{~mm}$ lens provided images of $161 \times 129 \mathrm{~mm}$ or $84 \times 67 \mathrm{~mm}$, respectively. These provided interrogation areas (32 x 32 pixels) - and thus spatial resolution - of about $4 \times 4 \mathrm{~mm}$ or $2 \times 2 \mathrm{~mm}$ respectively. Typically, the delay time 
between laser shots within each pair, whose resulting images were used for the cross-correlation algorithm, was set at $25 \mu \mathrm{s}$, to ensure that particles moved through less then $25 \%$ of the interrogation area between each image. Initially, $50 \%$ overlap of interrogation areas was used $(75 \%$ for the smaller image area); this meant that velocity vectors were estimated in additional areas that overlapped the initial, abutting areas. Spatial resolution is not improved by this means, of course (values of vectors in the overlapping areas are precisely what they would be in the absence of overlapping), but the resulting additional vectors are more accurate than values that could be deduced by simple interpolation from values in abutting areas. Later analysis of the same data used the 'high accuracy, adaptive algorithm', in which iterative changes and refinements in the interrogation areas are made so as to reduce bias errors due to peak locking and/or particles leaving the area in the first image before the second shot is obtained. This produced much smoother Reynolds stress data which were generally in good agreement with HWA data (see below) in regions where HWA was viable.

Most data were obtained with image planes parallel either to the $x-z$ or the $x-y$ planes. In the former case, the laser sheet was projected from above the tunnel and was coincident with the valley's spanwise centreline, whilst the camera was mounted outside the corner fillet, which had a special window inserted in it. For views in the horizontal plane, the positions of the camera and laser head were essentially transposed. Particles of average size around $2 \mu \mathrm{m}$ were injected into the flow from a seeder placed downstream of the working section. Some degree of trial and error in the applied length of generator bursts was needed to ensure adequate particle densities within the working section.

As an indication of the level of agreement between HWA and PIV data, Figure 2 shows mean velocity profiles obtained over the centre-line of the valley (axis normal to the upstream flow) and at the downstream edge $-x / L=0.5$ and $x / L=1.0$ respectively. Note that the origin of the $x, y, z$ coordinate system is at the (top) leading edge of the valley. Over the valley centre, the velocities near the valley bottom (i.e. close to $z / H=-1.0)$ are clearly negative - reattachment of the separated shear layer occurs a little further downstream - so the single hot wire data were not obtained below about $z / H=-0.4$, where local turbulence intensities were in excess of $60 \%$. Note that even there, the HWA data are somewhat above those from the PIV, because rectification errors become significant once intensities exceed about $40 \%$ (for a single wire). Above $z / H=0$, however, the results are in reasonable agreement. At the trailing edge, HWA data are again somewhat in excess of the PIV results, even though at $z / H=0.1$, for example, the local intensity was only around $30 \%$. However, at this location there was a not insignificant 


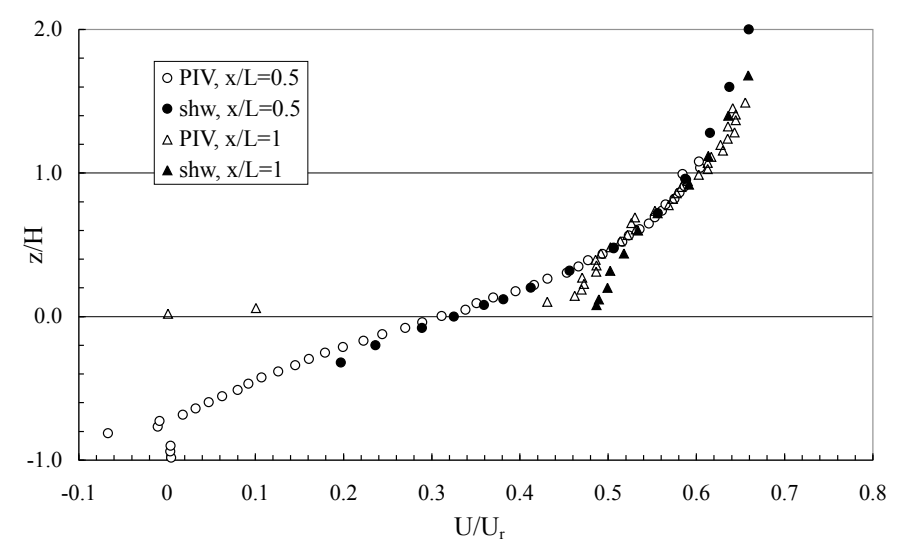

Figure 2. Comparisons between HWA and PIV data, for axial locations $x / L=0.5$ and 1.0.

vertical mean velocity component; a single hot wire (oriented spanwise as it was in this case) would measure the velocity vector magnitude in the vertical plane and thus over-estimate axial velocity. Crossed wire data (not shown) gave closer agreement. It should also be noted that close to the surface, the velocity gradients are high and even with the $2 \times 2 \mathrm{~mm}$ resolution one thus expects somewhat greater inaccuracies in the PIV data.

\subsection{The Simulated Boundary LAYeR}

A false floor whose surface was about $40 \mathrm{~mm}$ above the floor of the tunnel was inserted into the working section and was fitted at the front with nine Irwin (1981) spires $h=475 \mathrm{~mm}$ in height and spaced $h / 2$ apart in the spanwise direction. These each had a base width of $55 \mathrm{~mm}$ and a downstream central (axial) fin of length $120 \mathrm{~mm}$ at the base. The two spires furthest outboard from the tunnel centreline (i.e. one near each side-wall) had to be scaled down in size since they stood on the working section's corner fillets. Coarse-grained sandpaper with a mean grain size of about $1 \mathrm{~mm}$ was used for the rough surface; measurements (both with and without the spires) indicated that this had a $z_{o}$ of about $0.06 \mathrm{~mm}$. With a free-stream velocity of $30 \mathrm{~m} \mathrm{~s}^{-1}$ the roughness Reynolds number $\left(u_{*} z_{o} / \nu\right)$ was around four, ensuring that 


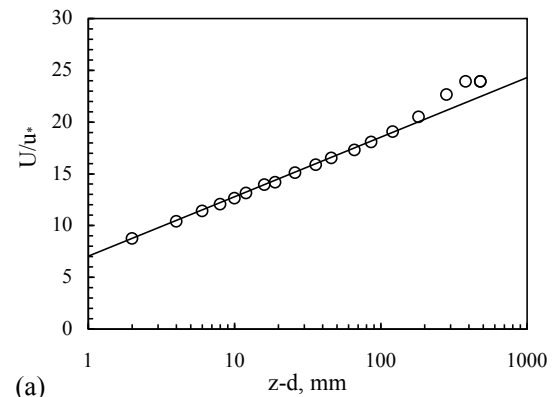

(a)

Figure 3. Mean velocity profile (a) and normalised turbulent stresses (b) in the reference boundary layer. In (b): $\overline{u^{2}}$, squares; $\overline{v^{2}}$, triangles; $\overline{w^{2}}$, circles; $-\overline{u w}$, crosses

the simulated boundary layer was fully aerodynamically rough; velocity and stress profiles at $4 \mathrm{~m}$ downwind of the spires are shown in Figure 3. The stresses have been normalised by the free stream velocity.

The shear velocity $u_{*}$ was deduced from the turbulence shear stress value in the near-wall region $\left(-\overline{u w} / U_{r}^{2}=0.0017\right)$. With the zeroplane displacement $(d)$ in the usual formulation of the log-law, $U / u_{*}=$ $(1 / \kappa) \ln \left[(z-d) / z_{0}\right]$, adjusted to give the best straight line - as shown in fig. $3 \mathrm{a}$ - the resulting value of $z_{0}$ was $0.06 \mathrm{~mm}$. $d$ was $1.0 \mathrm{~mm}$ and could of course have been partly accounted for by small errors in the vertical origin used for the probe height. Note that a good log-law region exists up to a height of about $100 \mathrm{~mm}$, below which the shear stress was roughly constant, as required (fig.3b). In fact, there is a hint of a peak in $-\overline{u w}$ just above $100 \mathrm{~mm}$; this peak is somewhat more pronounced further upstream and is thus evidence of a residual slow flow development with fetch. There is a corresponding slight kink in the $\overline{u^{2}}$ profile. The ratios between the various normal stresses and the shear stress are similar to those typical of a (rural type) atmospheric boundary layer. For example, Grant (1992) gives values for the ratios $\sigma_{u} / u_{*}$ and $\sigma_{w} / u_{*}$ of 2.29 and 1.14, respectively, in the near-surface layer (where $\sigma_{u}$ and $\sigma_{w}$ denote rms values of the axial and vertical velocity fluctuations; the data in figure $3 \mathrm{~b}$ suggest corresponding values at $z=20 \mathrm{~mm}$ of about 2.24 and 1.15 .

In addition, spectral data within the log-law region are similar to those given in the Engineering Sciences Data Unit (ESDU) data sheets for such an atmospheric boundary layer; fig.4a shows the raw $u$ - and $w$-velocity spectra at $z=50 \mathrm{~mm}$ and fig.4b is a normalised plot of the former. Note that the $w$ spectral values have been factored by $4 / 3$ so that collapse with the $u$ spectra would be expected in the inertial subrange provided the turbulence Reynolds number is sufficiently high. In fig. $4 \mathrm{~b}$ the data are compared with the standard ESDU spectrum and 

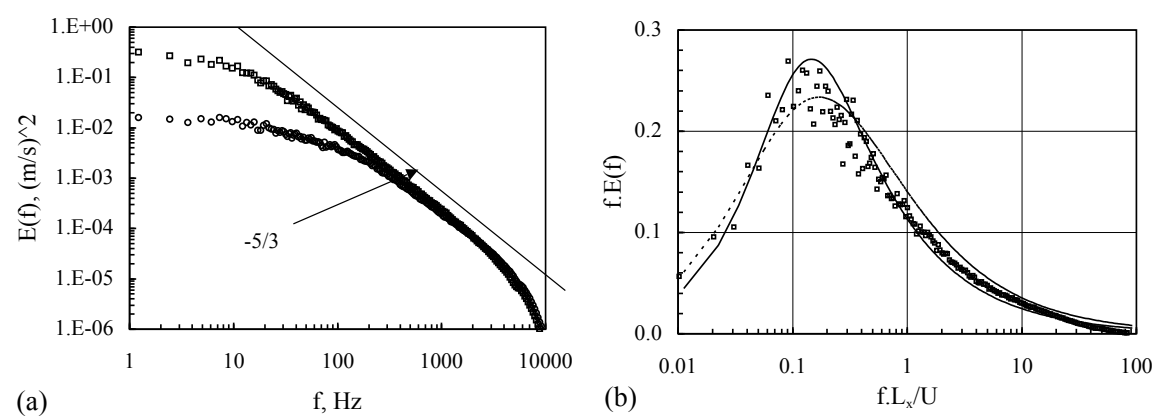

Figure 4. (a): Turbulence energy spectra: $E_{u}(f)$ (squares) and $E_{w}(f)$ (circles). (b), normalised $u$-spectra compared with the standard Engineering Sciences Data Unit (ESDU) spectrum (solid line) and Kaimal's ${ }^{[11]}$ spectrum (dashed line).

with the (only slightly different) spectral shape proposed by Kaimal \& Finnigan (1994), using an axial integral scale, $L_{x}$, of $170 \mathrm{~mm}$. Using the ESDU data, which provides a scale factor in terms of $L_{x}, z_{0}$ and height, it was found that in this case the apparent scale factor was around 550. This is within a factor of two of the geometrical scale of the valley itself. $H / z_{0}$ was 420 , compared with about 3200 in the field, but reductions in model $z_{0}$ to increase the former would have lead to a transitionally rough surface. Furthermore, since $H / \delta$ was about 0.062 compared with 0.026 in the field, increases in model $H$ would, whilst reducing the mismatch in $H / z_{0}$, increase the mismatch in $H / \delta$. Since, in addition, there was considerable uncertainty (and variability) in the precise value of $z_{0}$ in the field - changes in value by factors of two or three are easy to achieve with limited data! - we conclude that the model conditions were sufficiently similar to those in the field to allow sensible comparisons of the valley flows in each.

\section{Results and Discussion}

\subsection{Valley at $0^{\circ}$ to the approach Flow}

\subsubsection{The flow upstream}

We consider first the effect of the valley on the upstream flow. For a case in which the flow remains fully attached as it traverses the valley, linear arguments suggest that the pressure near the surface must first 
decrease as the flow enters the valley and thus the velocity will increase. The perturbation pressure field will undoubtedly extend upstream so one expects to see an increase in near surface velocities upstream of the valley. The effects are illustrated by the 'inverse' case of flow over an isolated hill, as discussed by Finnigan et al (1990), for example, who show an increase in surface pressure as the hill is approached (see below). For steeper hills or valleys, but still not steep enough (for the valley case) to promote separation, the effect will be greater. However, for valley slopes sufficiently steep to yield a separated region, although the streamlines near the leading edge might initially be more curved, the separation zone can crudely be considered as an extension to the upstream surface so that, overall, there will be much less downward movement of streamlines over the first part of the valley, at least for $z>0$. This will have the effect of reducing the decrease in pressure and corresponding increase in velocity upstream. This general behaviour is demonstrated by Snyder et al's (1991) experiments; figure 5a shows velocity profiles at the leading edge of the valleys, for the three cases (denoted V3, V5 and V8 in decreasing order of steepness). The data are shown as lines since, as explained earlier, it is the smoothed data (Busuoli et al, 1993) which are used here. Note in particular that although moving from V8 to V5 (increasing steepness) leads to increasing velocity perturbations, the trend is not continued for V3, because in this case the flow separates. This is all in accord with the expectations outlined above. Figure 5b shows the corresponding data from the present work. In both figures $z$ has been normalised using the appropriate boundary layer depth and the $z / \delta$ corresponding to $z=H$ is indicated, to give a feel for the valley depth (where, of course, $z=-H$ ). It should be noted that the flows in the outer layer of the boundary layers $(z / \delta>0.1$, say) are significantly influenced by the nature of the boundary layer simulation technique, which was quite different in the two experiments. The present spire technique is probably superior to the simple spanwise fence used by Snyder et al in terms of producing a more normal mean velocity profile, having a significant wake component in the outer layer. This detail is not significant in affecting the dynamics of the flows within the valley, since in both cases $H<<\delta$.

Choosing $z / \delta=0.01$ as a representative height close to the surface, the steepest valley in the Snyder case (V3) leads to a $15 \%$ increase in velocity, whereas the present data suggest an increase of only about $8 \%$. On the other hand, choosing a fixed $z / H=0.1$ as an alternative representative height, V3 gives a $9 \%$ increase compared with $12 \%$ for the present case. That the difference is in the opposite sense is simply a result of the different $H / \delta$ in the two cases (and perhaps also because the Snyder boundary layer was relatively rougher than the present one), 
but it can be concluded that the present steep-sided valley, in which steady separation occurs, has a very similar effect on the upstream velocity field to that shown by the Snyder (V3) experiment.

As mentioned above, an isolated hill would in general have an opposite effect on the upstream flow - pressure initially increasing and velocity decreasing near the surface as the upstream edge of the hill is approached. There have been numerous physical and numerical experiments and analytical theories on flow over isolated hills but, no doubt for practical reasons, most of the attention has been concentrated on the nature of the flow near the crest - where the maximum velocity speed-up is found - and in the immediate lee-side wake. There is thus very little detailed data available on the way on which the hill affects the characteristics of the upstream flow. However, the experiments of Finnigan et al (1990) showed that at the upstream edge of an isolated hill of height $0.1 \delta$ the velocity 'speed-down' at $z / H=0.1$ (with $H$ here equal to the hill height) was about $13 \%$, similar to the levels of speed-up seen for the valleys discussed here.

From the point of view of sediment transport, changes in the surface stress are also important. Figure 6 shows normalised $-\overline{u w}$ profiles corresponding to the velocity profiles in fig.5. A number of remarks are pertinent. Firstly, note that, as Finnigan et al (1990) pointed out, it is important to consider stresses in a streamline coordinate system (rather than the measurement $x, y, z$ system). All the data in fig. 6a have been rotated into the local flow direction; the raw (unrotated) data for the V3 valley are also shown. In this case, the flow at $x / L=0$ had a maximum (downward) component corresponding to a $4^{\circ}$ streamline angle and this clearly makes a significant difference to the shear stress values. The
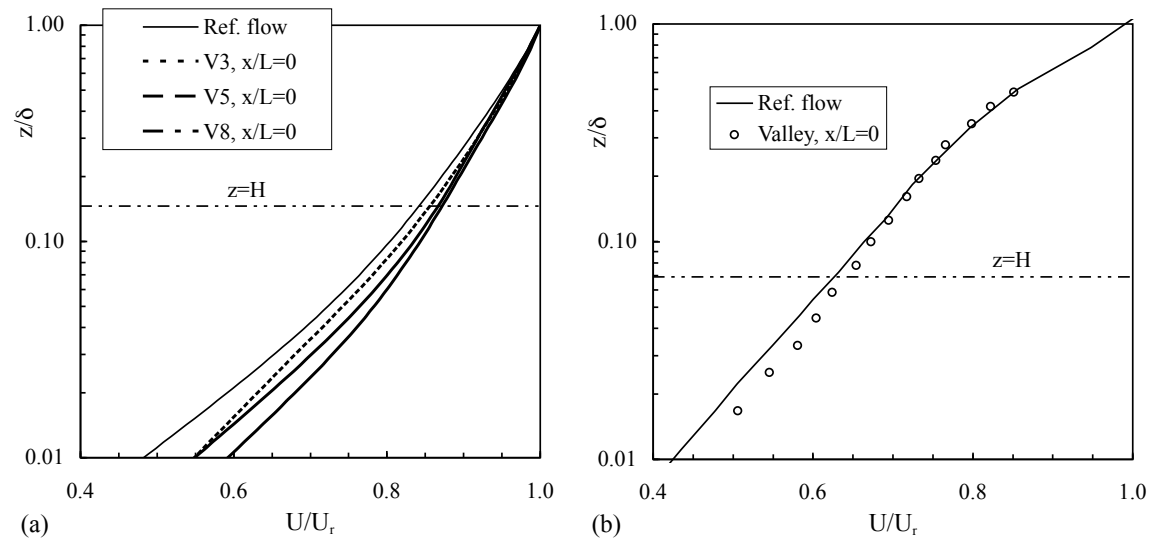

Figure 5. Mean velocity profiles at the valley leading edge, compared with reference profile in the absence of the valley. (a): Snyder et al (1991).; (b): present case. 

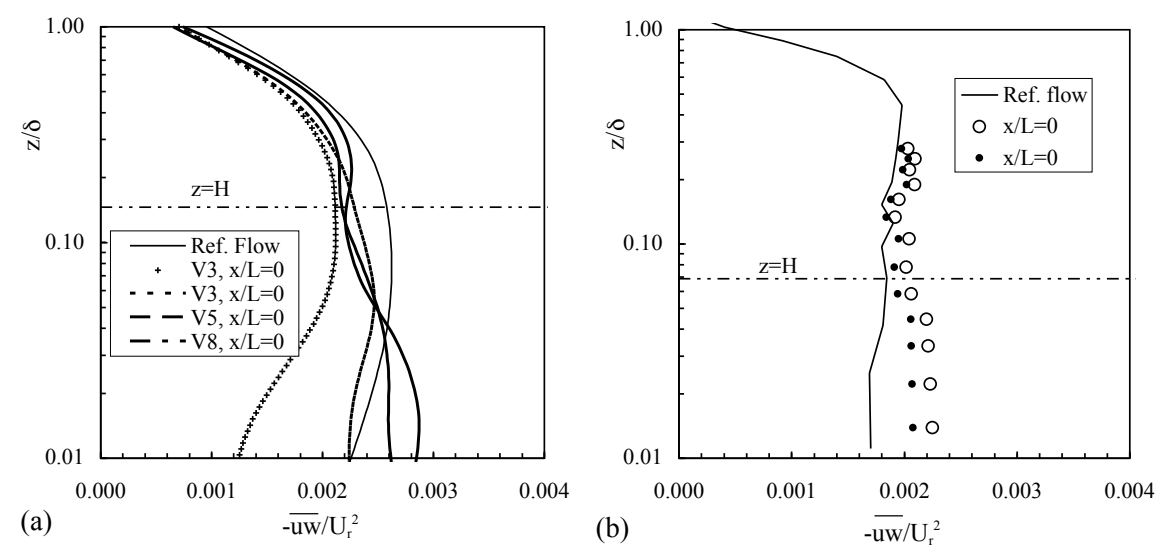

Figure 6. Shear stress profiles (in streamline coordinates) at the valley leading edge, compared with reference profile in the absence of the valley. (a): Snyder et al ${ }^{[5]}$, symbols refer to raw (unrotated) data for V3; (b): present case, solid symbols refer to raw (unrotated) data.

other two valleys had much smaller flow angles (at $x / L=0$ ) and the differences between the raw and rotated stresses are consequently much smaller.

Secondly, it is clear that in all cases (except the Snyder V3) the shear stress rises near the surface, as anticipated by linear theories (like those following Jackson \& Hunt, 1975). However, the rises do not follow the trend that might at first be anticipated. In line with the increased velocity perturbation as the valley steepness rises (V8 to V5 in fig.5a), one would expect a larger perturbation in $-\overline{u w}$ for V 5 than for V8, but the reverse is the case. Now previous work on flow over hills has shown that the streamline curvature affects the turbulence structure. In the case of hills, linear theory suggests that the shear stress will fall near the upwind edge of the hill. But the concave (i.e. destabilising) streamline curvature, which is not accounted for in linear theory, will tend to enhance the turbulence. Finnigan et al (1990) found that in their case the two effects roughly cancelled, so that there was virtually no change in shear stress by the upstream edge. Castro \& Wiggs (1994) found exactly the same for three-dimensional flow over a dune. In the present case, however, because the streamline curvature is significantly lower than in these hill cases, its affect, which is in these valley cases stabilising, is not strong enough to counterbalance entirely the expected change in shear stress. But since the curvature is undoubtedly greater for V5 than for V8 it is sufficient to reverse the expected trend from the shallowest valley (V8) to the somewhat steeper one (V5). This increasing effect of curvature is even more marked for 
the steepest valley (V3), so that the perturbation shear stress is for that case the smallest and, in fact, is negative.

Thirdly, note that the mean momentum equation suggests that at the surface the axial pressure gradient, $\partial p / \partial x$, must balance the vertical shear stress gradient, $\partial(-\overline{u w}) / \partial z$. So around the leading edge of the valley, where the former is negative, we might expect $-\overline{u w}$ to fall with height very near the surface. The data are not clearly consistent with that expectation, but it should be emphasised that crossed hot wire measurements near the surface, where (at least upstream of the valley) the turbulence levels are at their highest, are subject to rather higher uncertainties than elsewhere.

Fourthly, the present case yields a percentage increase in shear stress near the surface which is roughly similar in magnitude to the rise shown by the shallowest valley in the Snyder sequence (fig.6b, cf. V8 in fig. 6a). Although the mean velocity perturbation is most similar to the V3 case, as discussed above, the typical streamline curvature at the leading edge is very small - closest to V8 - so this result is consistent with the above stress perturbation arguments, in that curvature effects probably only have a marginal influence on the rise in shear stress expected on the basis of linear theory in the near-wall region.

\subsubsection{The flow over and within the valley}

The flow separates close to the leading edge of the valley, leading to a recirculation region within it. It is worth noting that initial experiments appeared to suggest no separation, in distinct contrast with the field situation where separation was very clear (Garvey et al, 2004). Close inspection of the wind tunnel model showed that, because the coarse-grained sandpaper had been simply 'wrapped' around the leading edge, the surface curvature there was (when scaled appropriately) much larger than in the field. This, along with the much smaller Reynolds number, was sufficient to prevent separation in the initial test. Since the intention was to model the full-scale flow, the model was modified slightly by cutting the sandpaper at the leading edge location to ensure a much sharper change in surface curvature and this led to the required steady separation, as occurred in the field.

Figure 7 shows vertical profiles of axial velocity at various axial locations within the valley $(0<x / L<1)$. The data were deduced from the time-averaged PIV vectors and the figures include data obtained at the leading and trailing edges, where the surface corresponds to $z / H=0$. In the central portion of the valley the surface corresponds to $z / H=-1$, but near the edges the valley side-slope means that the surface lies in the range $-1<z / H<0$ (as indicated on the figure). 

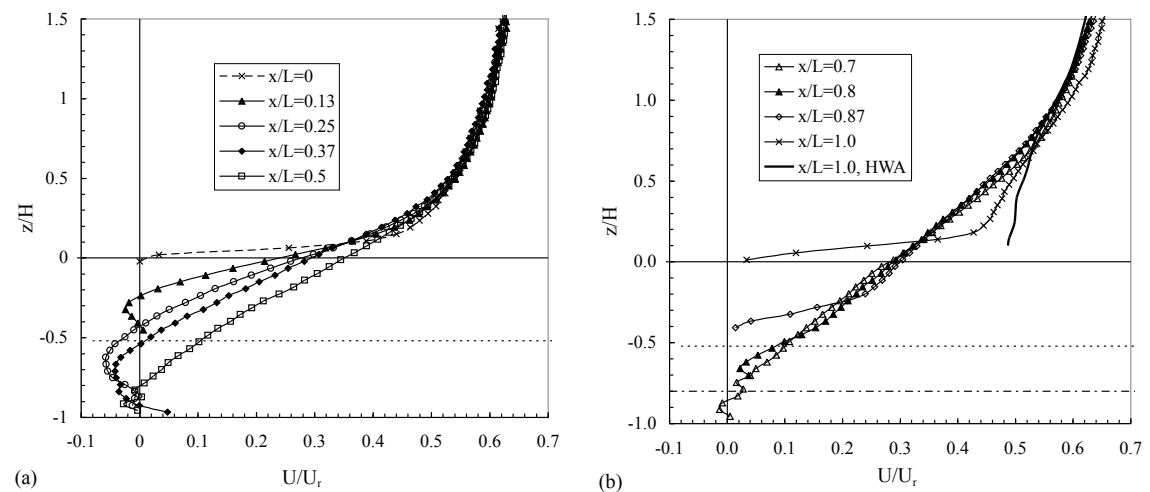

Figure \%. Velocity profiles, from PIV images. (a): $0<x / L<0.5$; (b): $0.5<x / L<1.0$; horizontal dotted and dash-dotted lines indicate the surface locations at $x / L=0.87$ (and 0.13 ) and $x / L=0.8$, respectively.

Regions of negative velocity are apparent up to at least $x / L=0.62$ (profiles near this location are not included in fig.7, for the sake of clarity) and mean reattachment must occur somewhere just prior to $x / L=0.7$. Note that the magnitude of the negative velocities nowhere exceeds about $0.06 U_{r}$. The accuracy of the data near the surface is more uncertain than elsewhere, because of unavoidable reflections, but the data do suggest that beyond reattachment and on the downstream slope of the valley, there are unusually deep regions near the surface in which the velocities are very low. Reattachment is of course a very unsteady process: two-dimensional bubbles often fluctuate in length by as much as $\pm 25 \%$. So one expects in this case that there would be significant periods of time during which the reattachment process occurs on the (downwind) sloping side of the valley. This may be the cause of the rather thicker 'dead air' regions than might otherwise be expected.

Figure 8 gives an overall impression of the mean velocity field within the valley. Simple colour shading has been used and it is clear that there is an extensive region within the valley where the velocity is below about $4 \mathrm{~m} \mathrm{~s}^{-1}$ (i.e. below about $0.12 U_{r}$ since $U_{r}=32 \mathrm{~m} \mathrm{~s}^{-1}$ ). The overlaps between the three images used to obtain this collage are evident, since there are slight discontinuities at the 'joints'. It should be emphasised that the $2 \mathrm{~m} \mathrm{~s}^{-1}$ integral steps between each colour inevitably tend to accentuate the lack of a perfect match. Note that the apparent presence of individual 'eddies' in the recirculating region must partly be caused by finite sampling size - recall that only 500 image pairs were used to obtain the statistics. However, there is rather more scatter in the mean velocity data when the values are close to 


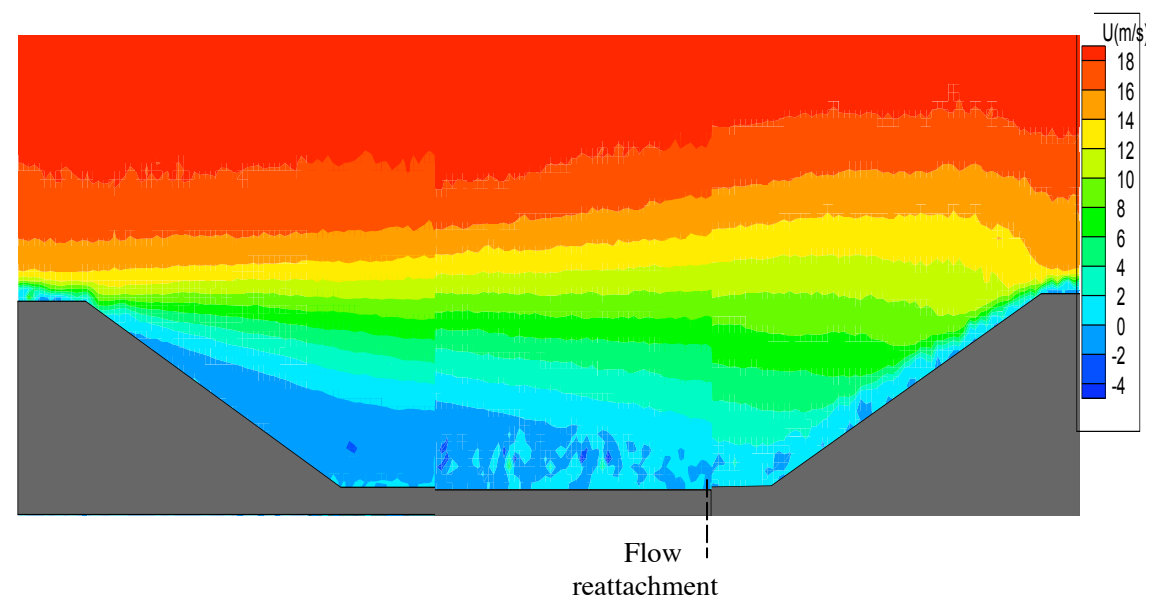

Figure 8. Velocity contours over the valley, from PIV images. Note the slight mismatch in vertical location of the three images which make up this picture.

zero (see fig.7) and some of this may be caused by inadequacies in the image correlation algorithm.

The turbulence stresses corresponding to the mean flow data of fig.7 are shown in Figures 9-11. At each axial location these have been produced by averaging (usually) the data at this location with those at the six nearest axial positions (three upstream and three downstream). Since the final interrogation area for each vector was about $1 \times 1$ $\mathrm{mm}^{2}$, this corresponds to a spatial average in the axial direction of about $3.5 \%$ of the valley width, which was considered small enough to ensure negligible errors arising from non-uniformity in the flow over that distance. The procedure significantly reduces scatter in the stress profiles.

Figure 9 shows that, not surprisingly, the axial turbulence stress develops significant peaks around the centre of the mixing layer which bounds the recirculation zone. The peak value reaches a maximum about half-way down the valley (around $\overline{u^{2}} / U_{r}^{2}=0.028$ at $x / L=0.5$, see fig. 9a) before slowly falling at locations further downstream. Note that for $x / L \leq 0.5$ and $z / H>0.5$ the profiles all collapse roughly to the values suggested by the hot wire data from the upstream flow. Further downstream, as the mixing layer grows in width, it begins to encroach on the upper level flow, so that at $x / L=1.0$, for example, the peak stress occurs around $z / H=0.7$. At this location, the hot wire data falls significantly below those from the PIV. This is undoubtedly a result of the significant errors arising in the former technique in locations of high turbulence intensity; axial intensities exceed $30 \%$ for $z / H \leq 0.5$ 
at $x / L=1.0$, for example. There is also a significant vertical mean velocity component near the surface at $x / L=1$ and this would further increase hot wire errors, since no attempt to orientate the probe into the mean flow direction was made.

The general behaviour of the axial stress profiles is mirrored by those of the vertical stress (fig.10) and the shear stress (fig.11), and the overall behaviour of the stresses is very similar to that in other well-documented, two-dimensional separated regions. (see, for example, Castro \& Haque (1986), where the inadequacies of hot wire anemometry in such flows are also discussed in some detail). A notable difference, however, is that the peaks in the $\overline{w^{2}}$ profiles occur significantly below those in the $\overline{u^{2}}$ profiles; this may be indicative of rather more shear layer 'flapping' than normally occurs - perhaps because of the relative proximity of the downstream valley slope, as mentioned earlier. A com-
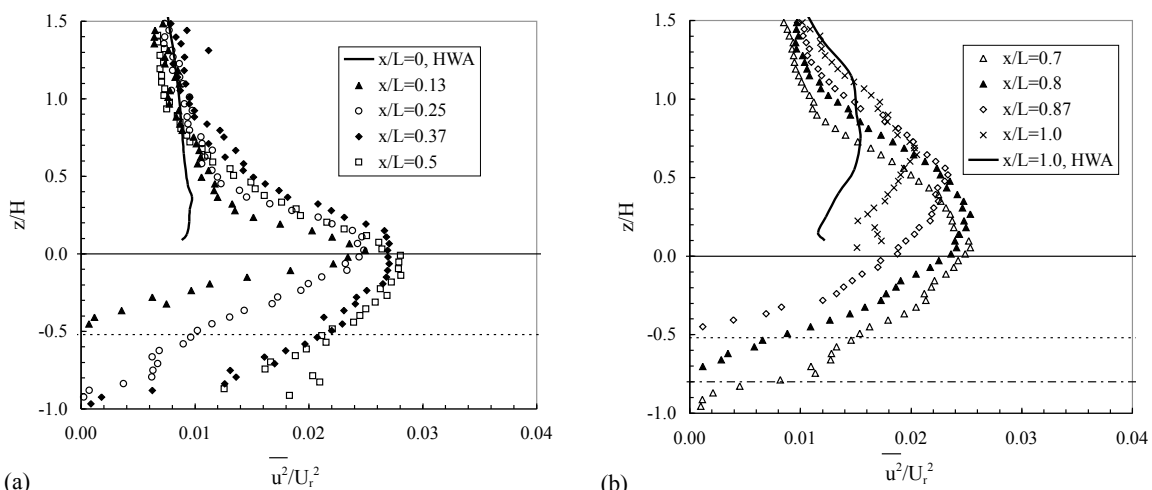

Figure 9. Axial stresses profiles. (a): $0<z / L<0.5$; (b): $0.5<z / L<1.0$; horizontal dotted and dash-dotted lines indicate the surface locations at $x / L=0.87$ (and 0.13 ) and $x / L=0.8$, respectively.
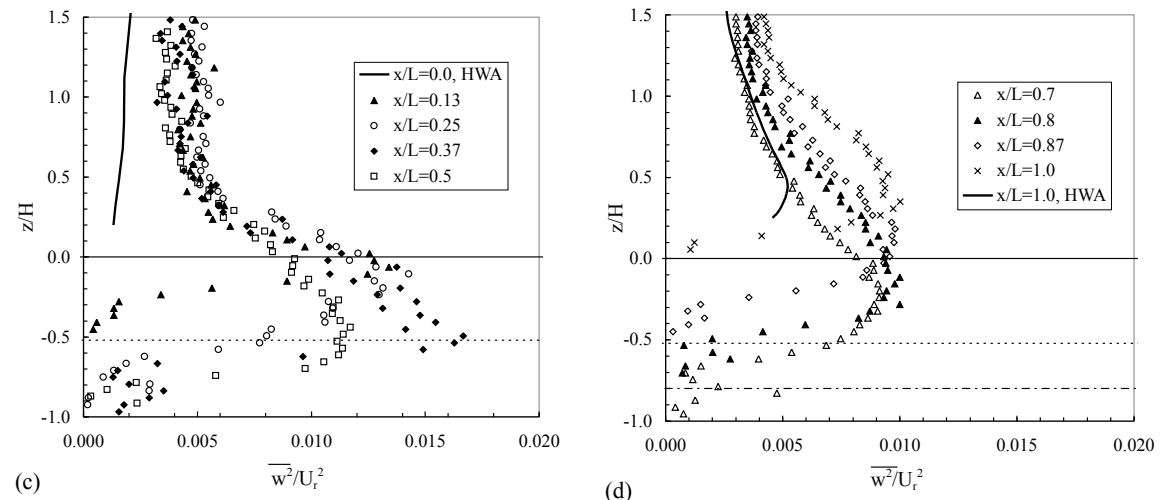

Figure 10. Vertical stress profiles over the valley. Other details as in Fig.9. 

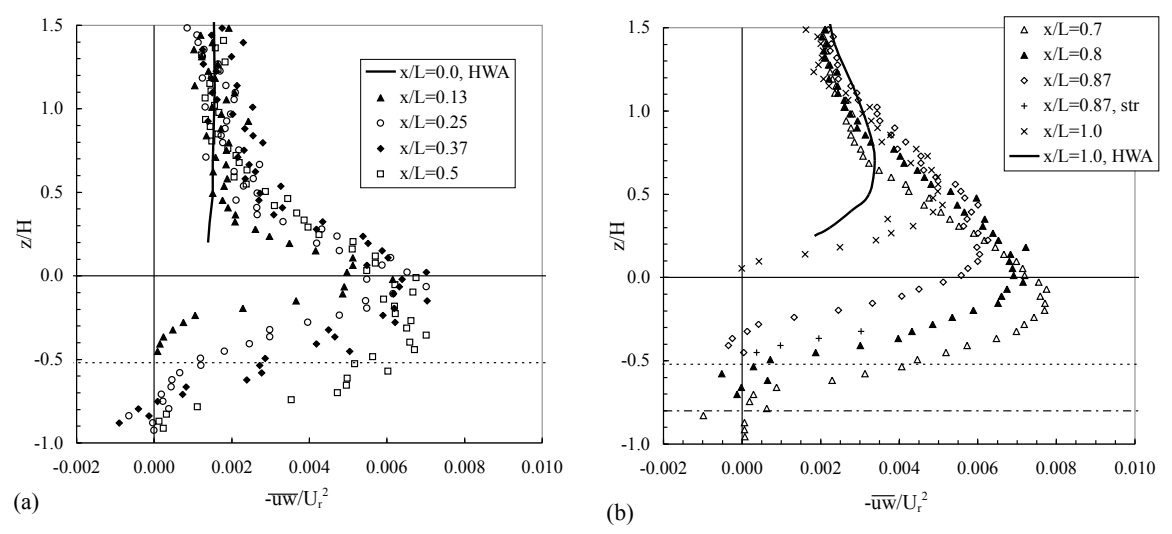

Figure 11. Shear stress profiles over the valley. Other details as in Fig.9, but note the additional data points for $x / L=0.87$, deduced by orientating the $x$ and $z$ co-ordinates into directions parallel and normal to the valley surface slope.

mon feature in all such flows, of course, is that the shear stress, $-\overline{u w}$, becomes very low near the surface. In fact, within the separated region one expects it to become negative, since the wall stress will be negative until the reattachment point. Negative values of $-\overline{u w}$ do indeed appear in the data (fig.11), corresponding roughly to locations where the velocity gradient is negative (see fig. 7), although both the scatter and the resolution prevent estimation of the wall stress by extrapolation to the surface. It seems possible, however, that the negative surface stresses within the recirculation zone may have magnitudes similar to the positive stresses in the reference boundary layer - see the data for $x / L=0.37, z / H<-0.75$ in fig.11a, a location about halfway to reattachment. This has implications for sand transport, which is driven by $u_{*}^{3}$ (according to most saltation models), so that local upwind transport within the valley is likely to occur, for wind conditions in which it occurs over the homogeneous surface upwind.

Negative values of $-\overline{u w}$ also occur on the downwind slope of the valley (see fig. $11 \mathrm{~b}, x / L=0.87$, for example), which seems inconsistent with (mean) attached flow there. But it should be emphasised that the stresses are here referred to a co-ordinate system aligned with the upstream flow, not the local flow direction. Just above the valley slopes, it would perhaps be more appropriate to use $x$ and $z$ axes parallel and normal to the slope. Fig.11b includes data for the shear stress rotated into that direction, for the first few points of the $(x / L=0.87)$ profile nearest the surface. This clearly removes the negative values, consistent with an attached flow up the slope.

It is worth noting, finally, how the present valley flow compares with that in the steepest of the Snyder valleys. Recall that the latter 
is steep enough to generate a large, mean separation region, just as in the present case. Figure 12 shows some profiles of mean velocity and axial stress at $x / L=0.62$ and 0.75 , compared with some of the present data. It is more appropriate here to use for the ordinate the distance from the local surface $\left(z^{\prime}\right)$, normalised by the boundary layer height $(\delta)$. Recall that, as noted earlier, there may be some uncertainty in the accuracy of the PIV data at the lowest levels; there is also some uncertainty about the Snyder data near the surface because of the details of the interpolation procedure used by Busuoli et al (1993) to generate 'smoothed' data. Nonetheless, it seems clear that in both cases the reversed flow region extends about $70 \%$ of the valley width from its leading edge. $H / \delta$ is different in the two cases (as well as the valley shape and the relative surface roughness) so one does not necessarily expect close agreement; indeed, $x_{R} / H$ (where $x_{R}$ is the distance to reattachment) is about 4 and 5.6 in the Snyder and the present cases, respectively, and this is arguably a direct result of the rougher boundary layer in the former case (see below). It is notable in fig.12b that the peak stresses are significantly higher in the Snyder case. (Rotating the stresses into streamline co-ordinates has very little effect on $\overline{u^{2}}$.) This difference is unlikely to be a result of any small difference in the extent of destabilising streamline curvature, as the $L / H$ and $L / \delta$ ratios are not too dissimilar in the two cases $(6,0.7$ and $8,0.5$ for Snyder et al, 1991, and the present case, respectively). It is probably simply a consequence of the generally larger turbulence levels in the reference flow - these are included in fig. $12 \mathrm{~b}$ and it can be seen that at $z^{\prime} / \delta=0.1$, for example, there is about an $80 \%$ difference in $\overline{u^{2}}$, reflecting the fact
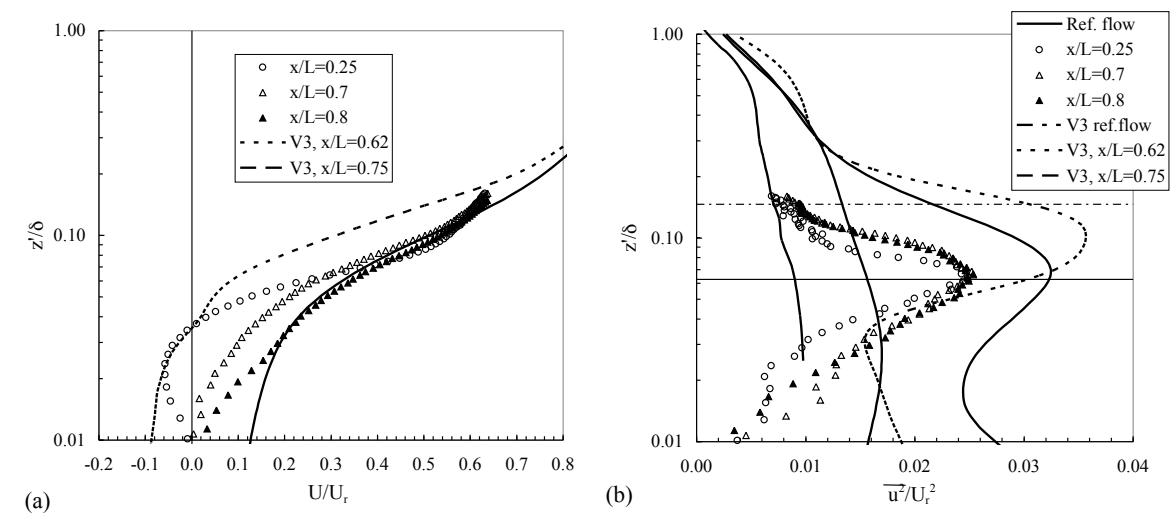

Figure 12. Mean velocity (a) and axial stress (b) profiles, compared with Snyder et al (1991) data. $z^{\prime}$ is measured from the surface in these plots and solid and dash-dot horizontal lines in (b) denote the valley top $(H / \delta)$ locations in the present and the Snyder experiments, respectively. 
that the Snyder boundary layer was significantly rougher than in the present case. It is also evident that the peak stresses occur noticeably below the level of the valley top, compared with the present case in which the peaks are around the valley top at all $x / L$. This is consistent with the separated region being relatively 'thinner' in the Snyder case - not surprising in view of the much smoother valley profile.

\subsubsection{The flow downstream of the valley}

Figures 13 and 14 show the development of the mean velocity and the turbulence stresses downwind of the valley. Hot wire data are shown, but the figures include some PIV data and the reference boundary layer profiles are also included. There is a significant difference between HWA and PIV data at $x / L=1.0$ - the trailing edge of the valley - as noted earlier. This difference (in mean velocity) is much smaller by $x / L=1.25$ (fig.13a). (PIV stress data for $x / L \geqslant 1.25$ are not shown, as it turned out that the quality for the most downstream set of images was much lower than in the upstream (three) sets, used for all preceding figures, and this led to large scatter in the stress data. The difficulty was almost certainly caused by a less satisfactory optical set-up.)

There is perhaps some evidence that the flow separates at the trailing edge; extrapolation of the shear stress data in fig.13b (for $x / L=1$ ), particularly in the case of the more accurate PIV data, certainly suggests that a change of sign may occur just above the surface, although the mean velocity appears to remain just positive. Because their valley was significantly smoother, there was certainly no separation on or beyond the downwind slope of the steepest Snyder valley but in the present case there is a discontinuous surface curvature at $x / L=1.0$, so one
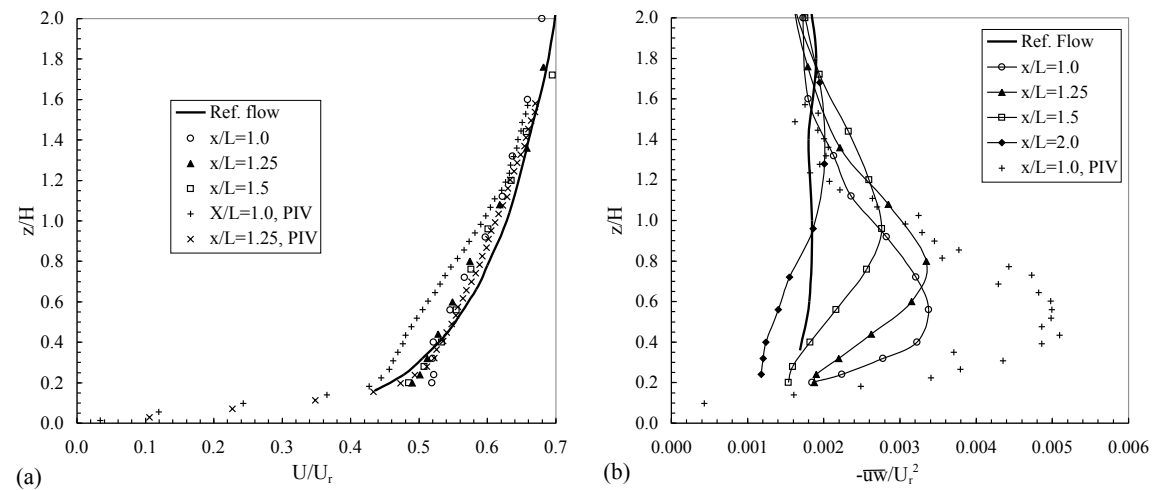

Figure 13. Mean velocity (a) and shear stress (b) profiles, downwind of the valley, compared with the reference profiles. 
might expect separation. However, in view of the uncertain accuracy very close to the surface it is not possible to be conclusive about this and, in any case, reattachment of any separated shear layer must occur very soon beyond $x / L=1.0$. The field data was also inconclusive in this regard (Wiggs et al, 2004), although there was some evidence of intermittent separation at least.

The stress profiles in figs.13b and 14 all indicate that the relaxation of the profiles towards the upstream conditions is characterised by profile peaks moving to higher elevations whilst reducing in magnitude with increasing downstream distance. One could view this downwind region as the valley 'wake' and the overall behaviour is very similar to the wake of isolated hills; studies by Finnigan et al (1990), Britter et al (1981), Castro \& Snyder (1982), for example, all show similar wake behaviour. Figures 13b and 14 might at first sight seem to suggest that the recovery of the stresses towards their reference levels is quite rapid. Note, however, that by $x / L=2.0$ both $-\overline{u w}$ and $\overline{w^{2}}$ have fallen below their reference levels - for $z / H<1.0$ and $z / H<0.75$, respectively (fig.13b and 14b). Although the boundary layer in the absence of the valley continues to develop somewhat over the fetch used for the valley, most of this development is in the outer part of the flow so that the turbulence stresses below $z / H=3$ are essentially the same at a location $2 L$ beyond the position of the valley's trailing edge. So the reference profiles shown in figures 13 and 14 are virtually indistinguishable from profiles taken at $x / L=2$ but in the valley's absence. There is previous evidence that in a boundary layer recovering after a region of separation - which is the situation here - the stresses do not return monotonically to the standard levels, but first undershoot the latter in the inner region. Recovery is, in fact, very slow (see Castro \& Epik, 1996, Cao \& Han-

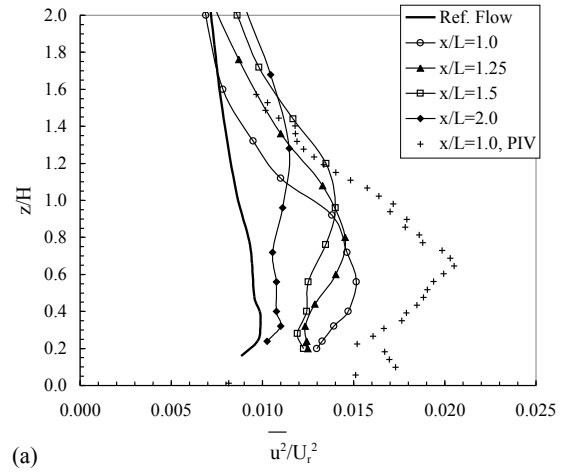

Figure 14. Axial (a) and vertical stress compared with the reference profiles.

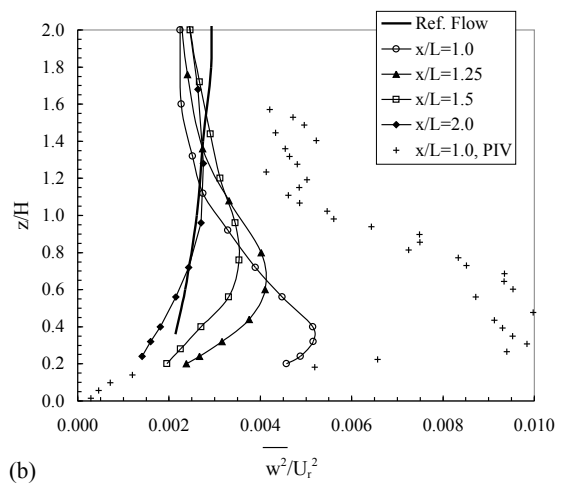

(b) profiles, downwind of the valley, 
cock, 2004). There is some evidence for similar behaviour in Snyder's steepest valley case. At $x / L=2,-\overline{u w}$ and $\overline{w^{2}}$ are below their reference levels for $z / H<0.2$ and $z / H<0.13$, respectively, although at both $x / L=1.5$ and $x / L=2.5$ the differences are marginal and probably within the experimental uncertainties. The region in which near-surface stresses are measurably below the upstream levels is therefore much more restricted than in the present case, starting perhaps just upstream of $x / L=2.0$, disappearing very soon and also extending much less far from the surface. These deductions are based on the smoothed data given by Busuoli et al (1993) and, quite apart from the interpolation uncertainties, are perhaps rather more uncertain in the downstream region because of the downstream development in the boundary layer in the absence of the valley - this was certainly greater than in the present case.

Field data are only available for $x / L=1.5$ and 3.5 so there is insufficient resolution to determine whether the stress profiles exhibited any non-monotonic behaviour during the recovery process. From a practical perspective, the major conclusion from the $x / L>1.0$ data is that the effects of the valley remain noticeable some way downstream - to at least one valley width from the trailing edge of the valley. The reduced surface stress (lower than in the absence of the valley in the range $1.5<x / L<2.5$ at least) would, if also present in the field, presumably lead to lower saltation rates from that region, compared with the conditions upstream of the valley, but there was no direct evidence of this (the appropriate region was not surveyed).

\subsection{Valley at $45^{\circ}$ to the approach Flow}

The general features identified and discussed above for the flow normal to the axis of the valley were found to be similar in many respects when the approach flow deviated from this direction. However, there were, not surprisingly, some significant differences and the more limited data presented in this section are chosen to highlight these differences. We start by discussing flow within the valley for it is here that the largest differences are apparent. Figure 15 shows velocity vectors on three horizontal planes below the valley top and one just above it. At $z / H=-0.9$ (fig. 15a) the illuminated plane was only $2.5 \mathrm{~mm}$ above the bottom surface of the valley and it intersected the valley slopes both upwind and downwind; these features together caused more surface reflection problems than for the usual vertical plane data so that a significant proportion of the vectors were invalid and have thus been 

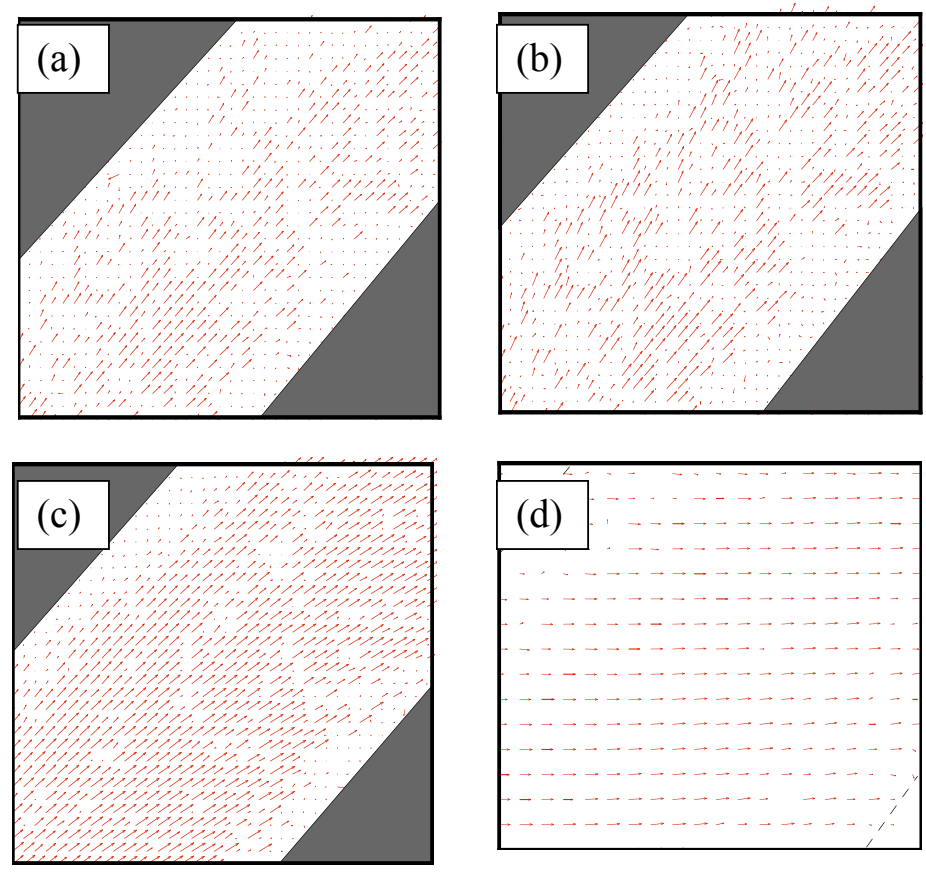

Figure 15. Mean velocity vectors from PIV images of flow within the $45^{\circ}$ valley at $z / H=:-0.9$ (a); -0.8 (b); -0.6 (c); 0.1 (d). The free stream flow is from left to right.

removed (their locations are shown by dots in the figures). There was a similar, but less serious, difficulty at $z / H=-0.8$ (fig.15b). At $z / H=$ -0.6 , just below the half-height plane within the valley, and at $z / H=$ 0.1 , just above the top of the valley, this problem was less acute and the general nature of the flow is quite clear (figs. 15c,d).

Near the floor of valley (fig.15a) the flow is aligned in the direction of the valley axis and this remains true even at $z / H=-0.6$ (fig.15c). In this latter position, however, it is evident that the flow in the downstream half of the valley has begun to be re-aligned into the free-stream direction. At $z / H=0.1$ this re-alignment is virtually complete, with the velocity vectors nearly parallel to the free stream velocity across the entire valley width (fig.15d); a noticeable deviation is, nonetheless, evident around the final $25 \%$ or so of the distance across the valley. It is interesting that in the first half of the valley the turning of the flow from the valley axis to the free stream directions occurs between $z / H=-0.6$ and 0.1 - compare figs. $15 \mathrm{c}$ and $15 \mathrm{~d}$ - whereas in the second half, this turning begins below $z / H=-0.6$ but remains incomplete at $z / H=0.1$. This may well be a result of the growth of the separated shear layer; the thickness of the region within which mean flow direction changes occur is likely to be related to the shear layer thickness. 
We define $L^{\prime}$ as the width of the valley in the $x$-direction (so that $L^{\prime}=\sqrt{2} L$, since the valley is at $45^{\circ}$ to the $x$-axis). The quantitative data indicate that at $x / L^{\prime}=0.5, z / H=-0.9$ the along-axis velocity has a magnitude of around $0.19 U_{r}$, which is much larger than the maximum near surface velocities (either positive or negative) in the case of the valley normal to the upstream flow (fig.7). This general channelling of the flow along the valley axis is very similar to the behaviour of flow over a linear dune (i.e. a small isolated hill) inclined to the upstream flow. Tsoar et al (1985) found that maximum deflections occurred in the lee of such a dune within the separated region and that the deflections decreased with height. This similarity is not really surprising and is undoubtedly a result of the pressure perturbations induced by the topography (Jackson, 1977).

A feature just about evident from fig.15a is that in the first half of the valley velocity vectors have a component normal to and towards the upstream slope, although the magnitudes are small compared with that of the along-axis component. This suggests that there is an attachment line somewhere around $x / H=0.5$, which was confirmed by vertical plane PIV data and is significantly upstream of the reattachment line in the case of the normal valley. Separation occurs along the leading edge, but the separation streamlines no doubt move helically, with the helix axis aligned roughly parallel to the valley axis. Being a fully three-dimensional flow, the attachment line is not of course formed by reattachment of the separation stream surface.

It is instructive to consider the extent to which the valley-axisaligned flow deep within the valley affects the flow for $z / H>0$. Figure 16 a shows $z$-profiles of flow direction in the horizontal plane at various axial positions. The data are from crossed hot wire measurements and therefore, near $z / H=0$, may not be very accurate - leading to
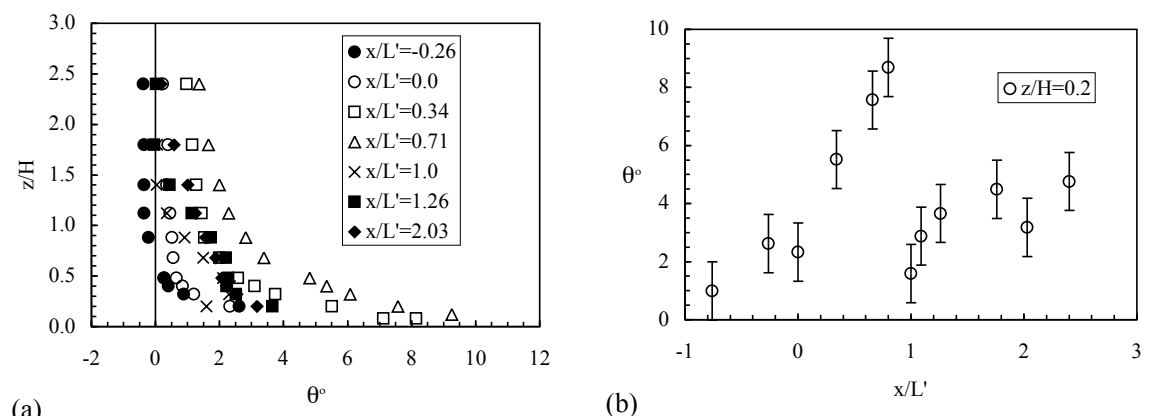

Figure 16. Flow angle in the horizontal plane, for the $45^{\circ}$ valley: (a) profiles at various $x / L^{\prime}$; (b) variation with fetch for $z / H=0.2$. 
uncertainties greater than those elsewhere. The latter may be some $\pm 1-2^{o}$. However, it is clear, first, that there is a small degree of flow turning upstream of the leading edge: the data at $x / L^{\prime}=-0.26$ show measurable deviations for $z / H<1.0$ although even at the leading edge $\left(x / L^{\prime}=0\right)$ this turning remains below about $2^{o}$ for $z / H>0.2$. These small changes are consistent with the finding that the axial velocity upstream of the leading edge (not shown) is hardly affected by the valley, so that even at $x / L^{\prime}=0$, the perturbations in mean velocity are scarcely noticeable. This is in distinct contrast with the situation for the normal valley, discussed in Section 3.1.1.

Figure 16a also shows that flow turning at $z / H>0$ increases with downstream distance, reaching a peak around $x / L^{\prime}=0.8$, before decreasing again towards the trailing edge and then, interestingly, increasing again further downstream. This is clarified in figure $16 \mathrm{~b}$, which shows the flow-angle changes with fetch, at a fixed height $(z / H=0.2)$. The fall prior to $x / L^{\prime}=1.0$ is quite rapid, whereas the subsequent rise is relatively slow. Only if the experimental uncertainties were unusually large for the data in the range $1.0<x / L^{\prime}<1.3$ could one surmise that there is, in fact, no fall and subsequent rise - the data could then be interpreted as suggesting simply a gradual recovery after the peak turning around $x / L^{\prime}=0.8$, which is certainly what might be expected intuitively. However, the full scale field experiments (Wiggs et al, 2004) provided some corroborating evidence for a fall in flow angle towards $x / L^{\prime}=1.0$ and a subsequent rise, so the phenomenon may well be real, although the reasons for it are obscure.

The freedom for the flow within and above the valley to be deflected sidewise leads to much smaller changes in the turbulence than in the
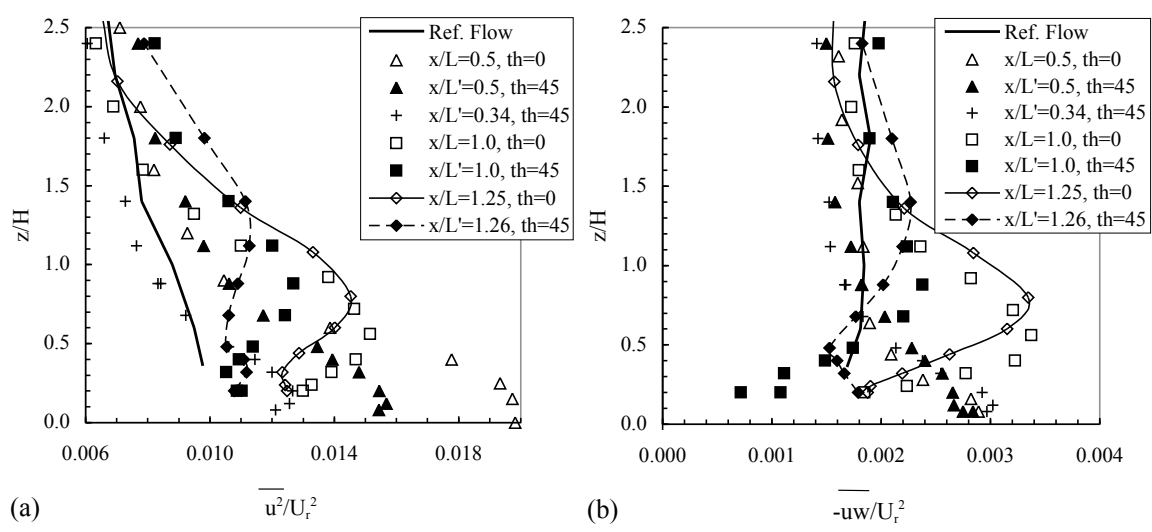

Figure 17. Turbulence stress profiles for $0^{\circ}$ and $45^{\circ}$ valleys: (a) $\overline{u^{2}}$ (note the false origin on the abscissa); (b) $-\overline{u w}$. 
normal valley case. This is illustrated in Figure 17, which shows profiles of $\overline{u^{2}}$ and $-\overline{u w}$ at various axial stations. The data are restricted to $z / H>0$ and only hot wire data are shown. As noted earlier, these are low compared with PIV data where both are available, but the trends are clear. Half way across the valley $(x / L=0.5)$ the peak $\overline{u^{2}}$ for the $45^{\circ}$ valley is only some $75 \%$ of its value for the normal valley. In terms of absolute distance, $x / L=0.5$ for the normal valley is equivalent to about $x / L^{\prime}=0.34$ for the $45^{\circ}$ case, so data for this location are included in the figure and indicate even smaller peak axial turbulence. In the case of the shear stress, the peak values seem rather similar between the two cases, at least at $x / L=0.5$. This quantity is particularly sensitive to flow direction and we have not rotated the coordinates in the $x-y$ plane, but that would lead to a reduction in the major shear stress component. By $x / L=1.0$, where the flow direction is roughly aligned with the upstream flow (fig.16b), the smaller peak shear stress in the $45^{\circ}$ valley case is clear and the axial turbulence, likewise, shows a much smaller perturbation from the reference flow than in the normal valley case. Some of the differences in the stresses between the normal and the $45^{\circ}$ cases may simply be that in the latter the upstream flow essentially sees a wider and less steep valley than in the former. Nonetheless, the 'freedom to be deflected sideways' is likely to provide the dominant reason for the smaller perturbations in the $45^{\circ}$ case.

Not surprisingly, the recovery of the turbulence downstream of the valley is more rapid than it is for the normal valley case. Figure 17 includes data for $x / L=1.25$ (highlighted by smooth curves) and it is clear not only that the peak stress levels are significantly lower in the $45^{\circ}$ valley case, but also that their heights above the surface are greater than for the normal valley. This is not simply because $x / L^{\prime}=1.25$ is further from $x=0$ than in the normal valley case. The general conclusion is clear: when the flow approaches the valley at $45^{\circ}$, the perturbations to the turbulence stresses are significantly reduced from what they would be for a normal approach flow. It seems likely, although we have no evidence to test it, that this reduction would be monotonic as the flow angle deviates from zero.

\section{Final Comments and Conclusions}

Our results have been shown to be generally consistent with those of Snyder et al (1991), for flow over smooth valleys. More detailed analyses of these latter results than have previously been reported have shown 
that, in particular, the flow over the present, sharp-crested valley is not too dissimilar to that over Snyder's steepest valley. In both cases, mean flow separated regions exist and turbulence levels in the elevated mixing layer and thus in the latter part of the valley and downwind of the trailing edge are very much higher than in the upstream boundary layer. These perturbations take a considerable distance to die away downstream; indeed, there is clear evidence (in the present case) of non-monotonicity in this recovery process, as found in other situations of boundary layers recovering after separated regions. Recovery is not complete even by eight valley depths downwind of the trailing edge and, within the recovery region, the surface stress is considerably lower than in the reference boundary layer. Within the valley, although the magnitude of the negative velocities occurring in the separated region nowhere exceeds about $0.06 U_{r}$, there can be significant (negative) shear stresses, implying that surface stress can reach magnitudes similar to those in the upstream boundary layer.

There are, however, some differences between the two cases and these are the direct result of the rather different geometries. A sharp leading edge, typical of desert valley situations, ensures immediate separation so that there are also some differences in the extent of the upwind influence of the valley. In the present case, both the mean velocity and the shear stress increase in the near-wall region prior to separation, by amounts which might be anticipated to be roughly in accord with linear theory; certainly, the reduction in mean velocity is very similar to the rise found near the upstream edge in isolated hill experiments (e.g. Finnigan et al, 1990), which themselves generally show good agreement with linear theory (e.g. Jackson \& Hunt, 1975) for the maximum speed-up near the hill crest. On the other hand, the trends in the various Snyder cases are more subtle, requiring consideration of the effects of streamline curvature. Downstream of the valley, recovery may be rather more rapid in the Snyder cases, probably because of the relatively smaller perturbations to the turbulence and the rougher upstream boundary layer.

With the upstream flow approaching the valley at $45^{\circ}$ to its axis, it has been shown how the flow is turned and channelled by the valley, so that near the floor of the valley the mean flow is dominated by a component along the valley axis. Whilst separation still occurs, it is much less extensive. The turbulence perturbations are much less severe, despite the additional mean strains generated by turning in the horizontal plane and, consequently, flow recovery downwind is rather more rapid.

Limited comparisons between the wind tunnel and field measurements have been presented elsewhere (Garvey, 2003) and we have al- 
ready made some further comments above. However, it is appropriate finally to highlight some of the major comparative results, if only to validate the use of wind tunnel models to explore the full-scale situation. We show, therefore, in Figures 18, fractional speed-up ratios at a position close to the surface for both field and model situations and for normal and $45^{\circ}$ valley orientations. The speed-up ratio is defined in the usual way as the ratio of percentage change in axial velocity from its upstream value at the same height. For the normal valley case (fig.18a) the overall agreement is very satisfying. Note that the small speed up prior to the leading edge occurs in both cases. Within the valley, the major difference is that reattachment occurs somewhat earlier in the field, where the measurements showed reversed flow close to the surface at $x / L=0.3$ but not at 0.5 . A possible explanation for this less extensive recirculation region may be the closer proximity of the downwind (forward-facing) slope: the field valley aspect ratio was about 7.7 where these measurements were made, compared with 8 in the model. The downwind slope, in affecting the pressure gradient within the valley (see McLean et al, 1996), may lead to mean reattachment earlier than would occur in its absence. On the other hand, in the classic case of flow down a rearward facing step, reattachment generally occurs some six step heights downstream although this is, of course, highly unsteady, with fluctuations in the attachment point location of at least $\pm 25 \%$. So the aspect ratio difference $(7.7 \mathrm{cf} .8)$ is unlikely to have a dominant effect. In any case, around the trailing edge of the valley, the field data shows similar speed-up ratios to those which occurred in the wind tunnel experiment. It is, perhaps, more likely that the shorter recirculation zone in the field was a result of relatively larger integral scales; these are strongly influenced by those longer time scale motions which are generally not possible to reproduce in the laboratory.
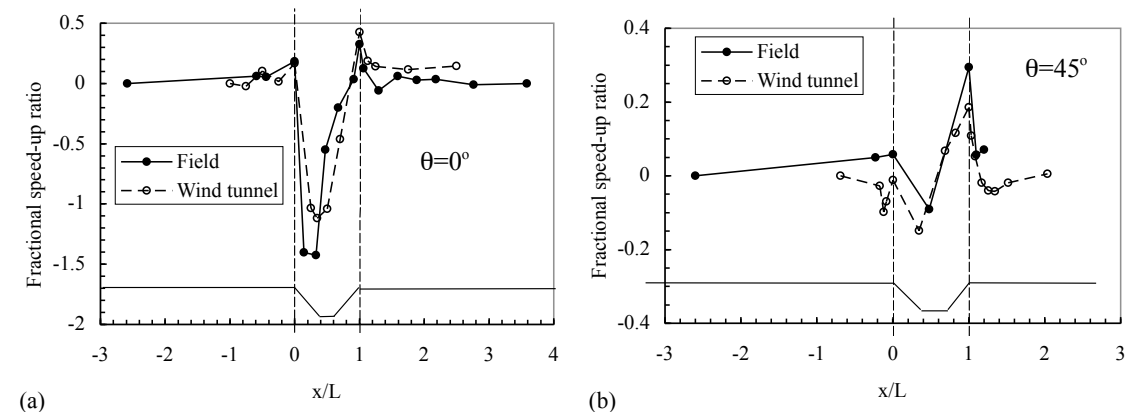

Figure 18. Fractional speed-up ratios at $z^{\prime} / \delta=0.005$ (wind tunnel) and 0.0056 (field) for approach angles of zero (a) and $45^{\circ}$ (b). 


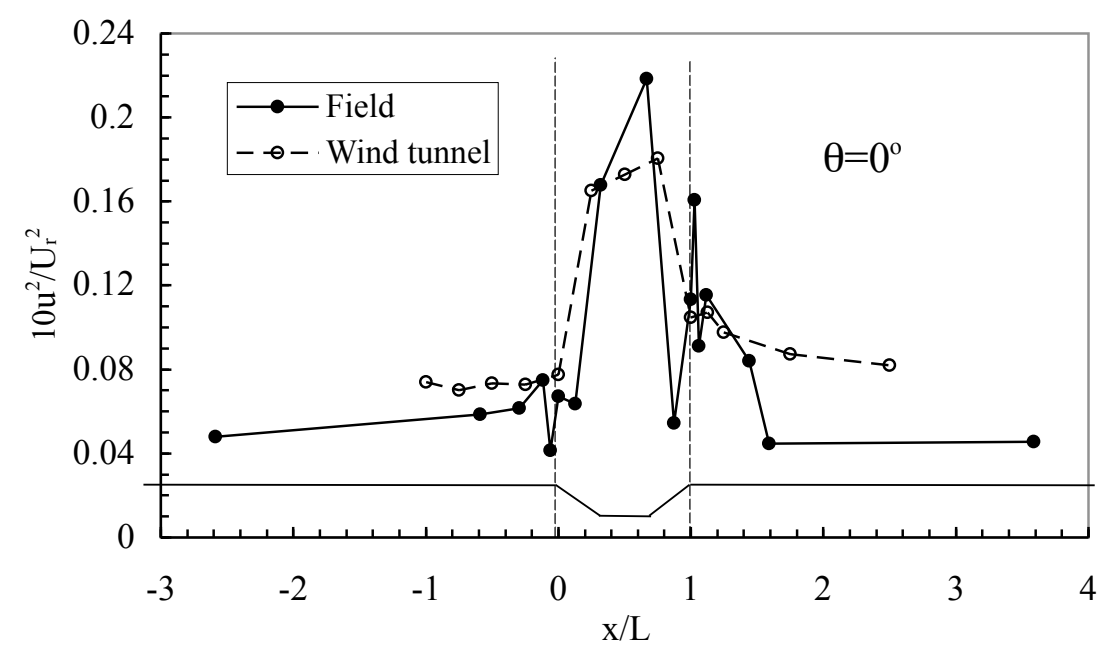

Figure 19. Variations in axial turbulence energy (same heights as in fig.18).

Stronger 'quasi-steady' fluctuations in the upwind flow, especially in the cross-stream direction, would tend to turn the flow more frequently into one at a non-normal angle to the valley axis, thus reducing the bubble length temporarily. However, no spectral data were obtained in the field, so this possibility must remain somewhat speculative.

For the $45^{\circ}$ valley (fig.18b) the overall changes in velocity are much lower (note the difference in axis scales between figs.18a and 18b) and, in the field, there seemed to be a slight increase in velocity towards the leading edge (as in the normal valley case). However, the field measurements are of total velocity magnitude (since cup anemometers were used) so take no account of the flow turning process described earlier. Within the valley, the uncertainties in the wind tunnel data may be significant (as discussed earlier, since these are from PIV images of planes very close to the surface). Overall, however, the behaviour is clearly very similar in the two cases.

Finally, figure 19 shows the variations in axial turbulence levels, measured at the same heights as the mean velocity data in fig.18. Again, the overall agreement is reasonable, although the upstream turbulence is rather lower in the field than in the wind tunnel. The peak values within the valley reach similar levels.

In conclusion, a number of features of the flow of a turbulent boundary layer over an incised valley have been identified and the results have been shown to be broadly consistent with those obtained in the associated field studies. They are also consistent with the very limited data already available in the literature and have been sufficient to 
emphasise the effects of differing valley geometry and wind orientation. It is anticipated that the results will be useful in formulating improved geomorphological models for desert situations affected by the presence of isolated valleys.

\section{Acknowledgements}

The authors are grateful to the Natural Environment Research Council, who funded the work under Grant no GR3/1210C and provided a studentship for one of us (BPG). Thanks are also due to the technical staff of the wind tunnel complex in the School of Engineering Sciences at Southampton, without whom this work would not have been possible. Particular thanks are due to Mr Geoff Baldwin for his help with the PIV data acquisition.

\section{References}

Baines, P. G.: 1995, Topographic effects in Stratified Flow, CUP, 482pp.

Britter, R. E. \& Hanna, S. A.: 2003, 'Flow and dispersion in urban areas', Ann. Rev. Fluid Mech., 35, 469-496.

Britter, R. E., Hunt, J. C. R. \& Richards, K. J.: 1981, 'Air-flow over a twodimensional hill: studies of velocity speed-up, roughness effects and turbulence', Quart. J. Roy. Meteorol. Soc., 107, 91-107.

Bullard, J. E. \& Nash, D. J.: 1998, 'Linear dune pattern variability in the vicinity of dry valleys in the southwest Kalihari', Geomorphology, 11, 189-203.

Busuoli, F., Trombetti, F. \& Tampieri, F.: 1993, 'Data sets for studies of flow and dispersion in complex terrain: II the "RUSVAL" wind tunnel experiment (flow data)', Tech.Paper 3, FISBAT-TP-93/1, 129pp.

Cao, C. \& Hancock, P. E.: 2004, 'Boundary layer development after a region of three-dimensional separation', Euro. J. Mech. B, Fluids, 23, 519-533.

Castro, I. P. \& Snyder, W. H.: 1982, 'A wind tunnel study of dispersion from sources downwind of three-dimensional hills', Atmos.Environ., 16, 1869-1887.

Castro, I. P. \& Wiggs, G. F. S.: 1994, 'Pulsed wire anemometry on rough surfaces, with application to desert sand dunes', J. Wind Eng. ES Ind. Aero., 52, 53-71.

Castro, I. P. \& Haque, A.: 1986, 'The structure of a turbulent shear layer bounding a separation region', J. Fluid Mech., 179, 439-468.

Castro, I. P. \& Epik, E.: 1996, 'Development of boundary layers after regions of separation', J. Fluid Mech., 374, 91-116.

Finnigan, J. J., Raupach, M. R., Bradley, E. F. \& Aldis, G. K.: 1990, 'A wind tunnel study of turbulent flow over a two-dimensional ridge', Boundary Layer Meteorol., 50, 277-317.

Garvey, B. G.: 2003, 'Wind flow over dryland valleys and implications for aeolian sediment transport', PhD Thesis, University of Sheffield, UK. 
Garvey, B., Wiggs, G. F. S., Castro, I. P. \& Bullard, J. E.: 2002, 'Wind tunnel investigations of flow dynamics within and around a valley', Proc. of 5th UK Conf. On Wind Engineering.

Garvey, B. G., Wiggs, G. F. S., Bullard, J. E. \& Castro, I. P.: 2004, 'Aeolian sediment dynamics in dryland environments: a field study of interactions with the fluvial system', Geomorphology, Vol.?

Grant, A. L. M.: 1992, 'The structure of turbulence in the near-neutral atmospheric boundary layer', J. Atmos. Sci., 49, 226-239.

Hunt, J. C. R., Leibovich, S. \& Richards, K. J.: 1988, 'Turbulent shear flow over low hills', Quart. J. Roy. Meteorol. Soc., 114, 1435-1470.

Irwin, H. P. A. H.: 1981, 'The design of spires for wind stimulation', J. Wind Eng. E Ind. Aero., 7, 361-366.

Jackson, P. S.: 1977, 'Aspects of surface wind behaviour', Wind Engineering, 1, 1-14.

Jackson, P. S. \& Hunt, J. C. R.: 1975, 'Turbulent flow over a low hill', Quart. J. Roy. Meteorol. Soc., 101, 929-955.

Kaimal, J. C. \& Finnigan, J. J.: 1994, 'Atmospheric Boundary Layer Flows: Their Structure and Measurement, OUP, New York.

Khurshudyan, L. H., Snyder, W. H., Nekrasov, R. E., Lawson, R. E., Thompson, R. S. \& Schiermeier, F. A.: 1990, 'Flow and dispersion of pollutants within twodimensional valleys: summary report on joint Soviet-American study', Tech. Rpt. EPA-600/3-90-025, Res. Tri. Pk., NC.

Kimura, F. \& Manins, P.: 1988, 'Blocking in periodic valleys', Bound. Layer Meteorol., 44, 137-169.

Lancaster, N.: 1988, 'Development of linear dunes in the southwest Kalihari, southern Africa', H. Arid Environ., 14, 233-244.

Louka, P., Belcher, S. E. \& Harrison, R. G.: 2000, 'Coupling between air flow in streets and the well-developed boundary layer aloft', Atmos. Environ., 34, 26132621.

McLean, S. R., Nelson, J. M. \& Shreve, R. L.: 1996, 'Flow sediment interactions in separating flow over bed-forms', In Ashworth P J (eds.), 'Coherent flow structures in open channels', Wiley, Chichester, pp 203-226.

Shankar, P. N. \& Deshpande, M. D.: 2000, 'Fluid mechanics in the driven cavity', Ann. Rev. Fluid Mech., 32, 93-136.

Sierputowski, P., Ostrowski, J. \& Cenedesa, A.: 1995, 'Experimental study of wind flow over the model of a valley', J. Wind Eng. Ind. Aero., 57, 127-136.

Snyder, W. H., Khurshudyan, L. H., Nekrasov, R. E., Lawson, R. E. \& Thompson, R. S.: 1991, 'Flow and dispersion of pollutants within two-dimensional valleys', J. Atmos. Environ., A25, 1347-1375.

Tsaor, H., Rasmussen, K. R., Sorenson, M. \& Willetts, B. B.: 1985, 'Laboratory study of flow over dunes', In Proc. of Int. Workshop on Physics of Blown Sand, Memoirs 8, Dept. of Theoretical Statistics, Aarhus University.

Weber, R. O. \& Kaufmann, P.: 1998, 'Relationship of synoptic winds and complex terrain flows during the MISTRAL field experiment', J. Appl. Met., 37: 14861496.

Wiggs, G. F. S., Bullard, J. E., Garvey, B. G. \& Castro, I. P.: 2002, 'Interactions between airflow and valley topography with implications for aeolian sediment transport', Physical Geography, 21, 366-380. 